\title{
Design of Real-time Image Processing System for Measurement and Calibration of PSP
}

\author{
MOHSEN DAVOUDI, AFSHIN HEIDARIAN \\ Department of Electrical Engineering \\ Imam Khomeini International University \\ University Blvd., Qazvin, \\ IRAN \\ davoudi@eng.ikiu.ac.ir
}

\begin{abstract}
The measurement of surface pressure in wind tunnel or in flight tests gives very important data for aerodynamics analysis. In fact, the pressure measurement gives valuable information about air flow phenomena such as shock, flow separation and boundary layer transition. In this paper a real-time image processing system for pressure sensitive paint (PSP) measurement and calibration has been designed using a calibrated pressure sensor. In the implementation of the system, TCL paper is used to luminaphore inside a windowed enclosure and a factory-calibrated pressure sensor for validation of measurement. The pressure measurement of PSP is based on the images acquired from a CCD camera sent to the MyRIO processor module in which the real-time image processing has been applied. The results are compared with the calibrated sensor from which the A and B coefficients of the Estern Volmer Equation have been calculated. During the several tests done for the pressure measurement, the accuracy of the PSP measurement has been calculated in this paper. Both normal and real-time image processing analysis have been compared as well.
\end{abstract}

Key-Words: Surface Pressure Measurement, Image Processing, Real-time Hardware, Pyrene Coating, Calibration

Received: September 17, 2019. Revised: January 2, 2020. Accepted: January 16, 2020. Published: January 25, 2020.

\section{Introduction}

The color pressure measurement method is a very powerful tool for the design of complex aerodynamic models, giving the designers a much deeper and better understanding of the complex phenomena of air flow. The principal of using Luminescence materials in colorimetric measurement of pressure is the characteristic of these materials in interaction with oxygen, known as oxygen reduction [1]. The brightness variations of the sample surface are captured by a CCD camera, and then using the image processing the amount of brightness variation of each pixel has been measured. In fact, in this measurement method, each pixel on the surface will act as a pressure sensor, thereby a very high resolution pressure profile can be achieved.

PSP test has many advantages over pressure tapes for surface pressure measurement, without any changes to the measurement model a much higher resolution of surface pressure measurement will be attained. In addition, there is much lower cost as well as shorter implementation time comparing to common measurement methods in aviation industry [2]. Surface pressure measurement using pressure tapes requires hundreds of pressure bands.
Manufacturing and installation of pressure bands is time-consuming and costly in which the measurement resolution will be limited, and a large amount of surface pressure data will be lost.

To Perform the test and calibration procedure, a set of tools are required: a Chamber with a glass window, a CCD camera with USB port, a lamp with appropriate wavelength, pre-coated luminophore material sample, optical filters, a calibrated pressure sensor to determine the pressure at any instant (for calibration of the PSP measurement method). In this paper a graphical user interface software has been designed to obtain information from the calibrated pressure sensor and the CCD camera to apply the image processing in real-time in order to detect sample brightness changes and translate it to the pressure profile of the surface.

In this paper, an isolation chamber is designed and prototyped to allow uniform pressure measurement on the surface of the sample to be validated with an accurate calibrated pressure sensor as a measure of chamber pressure changes. Pressure sensor data has been transmitted to the computer via an interface board in line with the CCD camera frames data. A serial port (COM) data acquisition code is written 
alongside the image processing program to analyze the information in real-time in a MyRIO device of National Instruments that is specialized for real-time processes. The image processing program is designed to display the measured surface pressure at high resolution in a two-dimensional and threedimensional chart. Fig. 1 shows an overview of the PSP measurement system [13].

The structure of this paper is organized as follows: A review and modeling of the surface pressure measurement method is briefly described in section two. The third sections presents the design and implementation procedure of hardware and software and real-time implementation challenges. Section four describes the system calibration process. The results of the experiments and their interpretation are presented in section five as well.

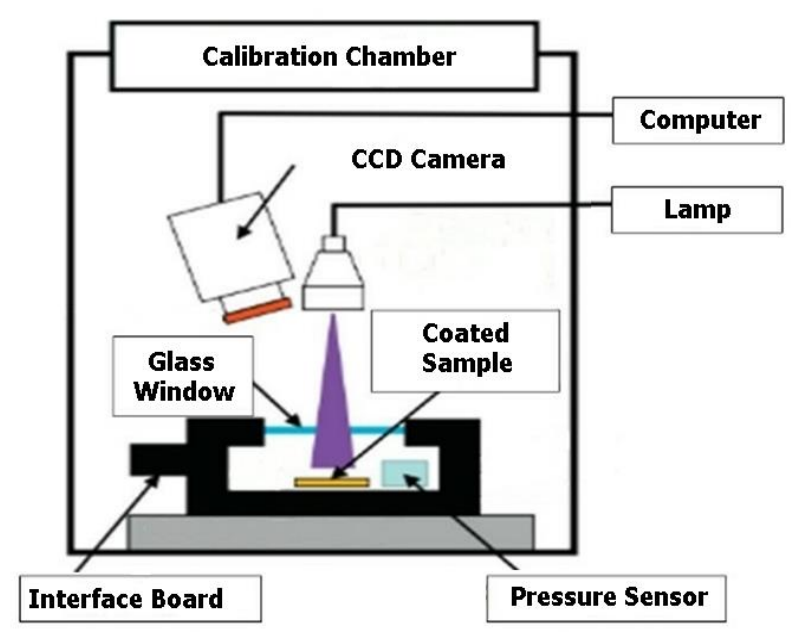

Fig. 1: Overview of how the chamber is positioned, and applied during the test calibration step.

\section{Modeling the PSP System}

An ideal pressure measurement system should be completely independent of temperature and not be affected from temperature changes. According to equation 1, a temperature-independent PSP system should have very low kinetic energy $E_{D}$ for the oxygen diffusion process. This process is represented by the $B_{\text {polymer }}$ in the Estern-Volmer equation. The Estern-Volmer coefficient should be very low. Since the Estern-Volmer coefficients are temperature dependent, and this is an inherent dependence, the PSP measurement system cannot be constant for different temperatures. Therefore, in an ideal PSP measurement system, the temperature assumes to be constant, to measure different pressures, and a different graph is drawn as the temperature changes [6].

$$
\begin{gathered}
B_{\text {polymer }}=B_{\text {polymer.ref }}\left(+\frac{E_{D}}{R T_{r e f}}\left(\frac{T-T_{r e f}}{T_{r e f}}\right)\right) \\
\frac{I_{0}(T)}{I(p . T)}=1+k_{s v}(T) p \\
I_{0}(T)=I(p=0 . T)
\end{gathered}
$$

In Equation 2, $I$ is the amount of illumination at zero atmospheric pressure conditions, and according to Equation 4 , the $\mathrm{k}_{\mathrm{sv}}(\mathrm{T})$ coefficient is related to $B_{\text {polymer }}(T)$ and $A_{\text {polymer }}(T)$ coefficients.

$$
g(T) \frac{I_{\text {ref }}}{I}=A_{\text {polymer .ref }}+B_{\text {polymer .ref }} \frac{P}{P_{\text {ref }}}
$$

If the coefficients are equally dependent on the temperature, the Estern-Volmer $\mathrm{k}_{\mathrm{sv}}(\mathrm{T})$ coefficient will not be temperature dependent [1].

Therefore, for an ideal PSP, the Estern-Volmer $\mathrm{k}_{\mathrm{sv}}(\mathrm{T})$ coefficient in Equation (4) will be more than $A_{\text {polymer }}(T)$ and $B_{\text {polymer }}(T)$ while the coefficients are independent of temperature. As a result, the Estern-Volmer equation in aerodynamic applications is written as equation 5 .

$$
k_{s v}(T)=\left[\frac{B_{\text {polymer }}(T)}{A_{\text {polymer }}(T)} * \frac{1}{p_{\text {ref }}}\right]
$$

The function $\mathrm{g}(\mathrm{T})$ is defined as equation 6 .

$$
\begin{gathered}
g(T)=\left[1+\frac{E_{D}}{R T_{r e f}}\left(\frac{T-T_{r e f}}{T_{r e f}}\right)\right]^{-1}= \\
{\left[+\frac{E_{r r}}{R T_{r e f}}\left(\frac{T-T_{r e f}}{T_{r e f}}\right)\right]^{-1}}
\end{gathered}
$$

For an ideal PSP test, Estern-Volmer equation enjoys a good homogeneity, and this is not changed in variables $g(T) \frac{I_{r e f}}{I}$ at different temperatures. The effect of temperature on the PSP is determined by the factor $g(T)$. This factor simplifies the investigation of the influence of temperature on the PSP test to some extent [7]. 


\section{Implementation of PSP measurement method}

\subsection{Sample coverage}

The luminophore materials and the retaining layer used in the PSP test plays an important role in the test results, and can affect test properties such as response time, temperature dependence, moisture dependence and the stimulated luminophore material brightness [3]. The measurement system of this paper uses pyrene luminophore material. Pyrene material has very low temperature sensitivity, better accessibility and lower price than other luminophore materials. It can measure the pressure range between $800 \mathrm{mbar}$ to $1100 \mathrm{mbar}$. The excitation wavelength of this luminescence material is $320-340 \mathrm{~nm}$. Pyrene is produced as a green powder that emits a pale blue after stimulation. Another material used in this test is the perylene material with an excitation wavelength of $330-450 \mathrm{~nm}$. The appearance of this luminophore is a brown powder. By combining $0.05 \mathrm{mg}$ of pyrene luminophore or $0.025 \mathrm{mg}$ of perylene luminophore and $10 \mathrm{cc}$ of dichloromethane solvent, the case solution is ready for coating. The sample has been dipped in paint for coating. Due to the fact that the dichloromethane solvent evaporates quickly, the test must be carried out at the appropriate speed so that the solvent in the evaporation solution does not evaporate. On the other hand, special attention should be paid to safety when performing this operation because of the toxicity of the chlorine solution [4].

The reaction of the luminescent material with oxygen reduces the excited electrons, which in turn reduces the luminescence action, known as phenomenon of reduced oxygen exposure. According to Henry's law, the concentration of oxygen in the retaining layer is proportional to the amount of pressure the gas exerts on the surface. In other words, as the pressure increases, more of the luminophore molecules lose their radiation or the process of lowering the luminescence with oxygen increases, so the surface brightness decreases, which means that the pressure on the surface is inversely correlated with the brightness level. The relationship between surface brightness and the amount of oxygen present on the surface is presented by the equation 7. This relationship has been derived from aerodynamic experiments, and is known as the Estern-Volmer equation [1].

$$
\frac{I_{r e f}}{I}=A+B \frac{P}{P_{\text {ref }}}
$$

So that, $I_{\text {ref }}$ and $p_{\text {ref }}$ are the luminescence brightness and the pressure level, respectively, in the reference conditions. A and $\mathrm{B}$ are coefficients of Estern-Volmer equation, due to the influence of temperature on the measurement during calibration, can be achieved.

Table 1: Specifications of pyrene material [6]

\begin{tabular}{|l|l|}
\hline Name & Pyrene \\
\hline Other name & Benzo $[c]$ phenanthrene \\
\hline The chemical formula & $\mathrm{C}_{16} \mathrm{H}_{12}$ \\
\hline Molar & $202.26 \mathrm{~g} / \mathrm{mol}$ \\
\hline Solubility & $0.135 \mathrm{~g} / \mathrm{mol}\left(25^{0}\right)$ \\
\hline Boiling point & $393^{0}(1013 \mathrm{hpa})$ \\
\hline Melting point & $148-150 C^{0}$ \\
\hline Flash point & $200-220 C^{0}$ \\
\hline
\end{tabular}

Theoretically, the ratio of brightness $\frac{I_{\text {ref }}}{I}$ can eliminate the effects of non-uniform brightness. Nonuniform illumination refers to changes that may occur due to inappropriate coating or uneven placement of luminophore materials on the surface. In typical tests, $I_{\text {ref }}$ is the surface brightness is when the wind tunnel is off and there is no wind, known as "Wind off". I is the amount of brightness when the tunnel is lit [12]. The pyrene material is purchased from the German Company "Merck", and general 
information on this material can be found in Table 1 [6].

\subsection{Excitation wavelength lamp and camera}

This measurement system uses a UV-A lamp to generate the excitation wavelength for the pyrene material. As mentioned, the excitation wavelength for the pyrene material is 320 to $340 \mathrm{~nm}$. The UVABLB lamps have a peak wavelength of $370 \mathrm{~nm}$, and have the range needed to stimulate the pyrene and perylene material. This lamp is made by Philips Poland [6]. Also, a camera with a CCD sensor was used to capture and transmit light changes caused by temperature changes during the calibration process. The camera used in this measurement system is a Basler camera piA1600-35gm. This digital camera is capable of receiving high resolution images in low light, and connected to a computer via USB cable and transmits images in real time at $35 \mathrm{Frame} / \mathrm{s}$ for analysis [7]. The reason for using CCD cameras is their low noise and higher brightness sensitivity than CMOS sensor. The camera is also capable of capturing $2^{16}$ pixel depth.

\subsection{Calibration chamber}

In the calibration procedure of the PSP color tester, a metallic box is used in which the adjusting the pressure is possible as well as isolating the test environment from possible noise such as changes in light and temperature unintentionally [5]. The calibration chamber is made of stainless steel, which is welded by a MIG/MAG welding suitable for the construction, and welding of pipes and housing against air leakage. The compartment has a transparent Plexiglas window to allow the illumination of the coated sample to be visible, and captured by the camera outside the chamber. This transparent window should be designed to be opened so that the necessary equipment such as the sample to be tested, as well as the pressure sensor can be installed in the box. Fig. 2 shows a view of the designed chamber.

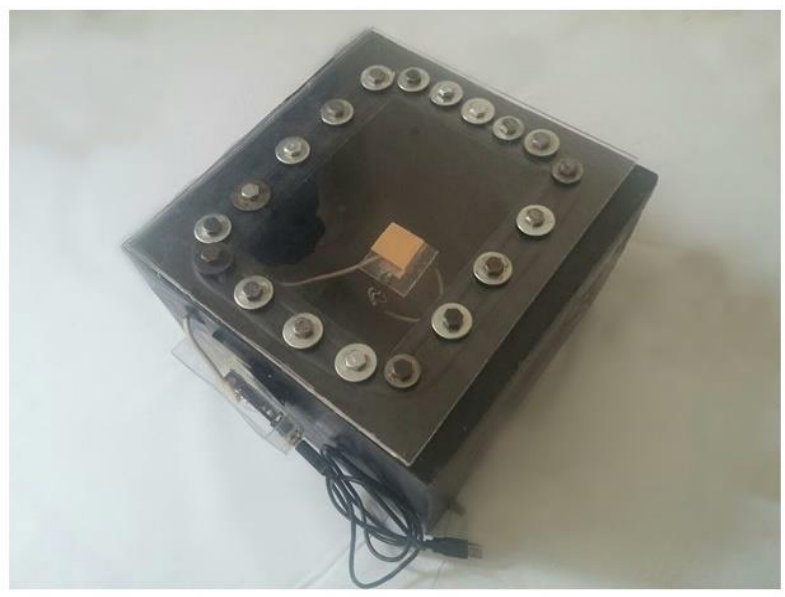

Fig. 2: An overview of the chamber designed for PSP testing

\subsection{Benchmark pressure sensor in calibration}

A commercial calibrated absolute pressure sensor was used to calibrate the data obtained from PSP tests. The BOSCH BMP180 Barometric Pressure Sensor Module is capable of measuring relative pressure in addition to absolute pressure. This module also has a temperature sensor and allows the temperature changes in the pressure test to be monitored with accuracy of $2^{\circ} \mathrm{C}$. The BMP 180 sensor is designed and manufactured based on piezoelectric resistance technology, can measure pressures between 300 and $1100 \mathrm{hPa}$ with absolute accuracy of $0.03 \mathrm{hPa}$ at a temperature range of -40 to $+85^{\circ} \mathrm{C}$ [8]. This technology has provided high accuracy, linearity and long lasting stability for this sensor. The sensor transmits pressure and temperature data in digital format $\left(\mathrm{I}^{2} \mathrm{C}\right.$ interface) to the microcontroller of the interface board, and from there to the computer via USB port. This sensor has a E2PROM memory where the calibration information is stored by the manufacturer precisely, and does not need to be recalibrated [8]. Fig. (3) shows the position of the calibrated pressure sensor module in the chamber. 


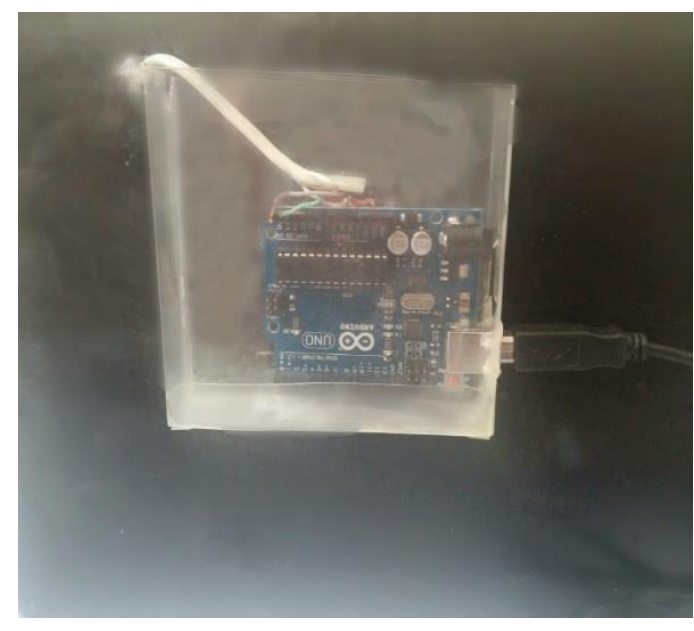

Fig. 3: Placement of the calibrated pressure sensor in the chamber

\subsection{LabVIEW data monitoring program}

After the hardware implementation of the PSP measurement system, software coding was performed to obtain the camera data and the calibrated pressure sensor. The code for the image processing program is written in LabVIEW and under the "VISA" Driver and Functions [9]. The main purpose of image processing in PSP testing is to measure the changes in brightness caused by pressure changes on the sample surface.

As stated, increasing the pressure on the surface of the coated sample increases the density of oxygen molecules on the surface, resulting in an increase in the oxygen reaction with the luminophore molecules and a decrease in brightness. In Fig. 4 the block diagram of the proposed image processing steps for calculation of the brightness and calibration has been presented.

As shown in Fig. 5, parts of the image that do not contain important information are removed before the image is processed, resulting in shorter computation time and higher frame rate, higher image processing accuracy and noise reduction.

After deleting the exterior areas, the image needs to be converted to Gray scale that only contains the "brightness" value required among the four pixel values of the image (green, blue, red, brightness) $[10,11]$.

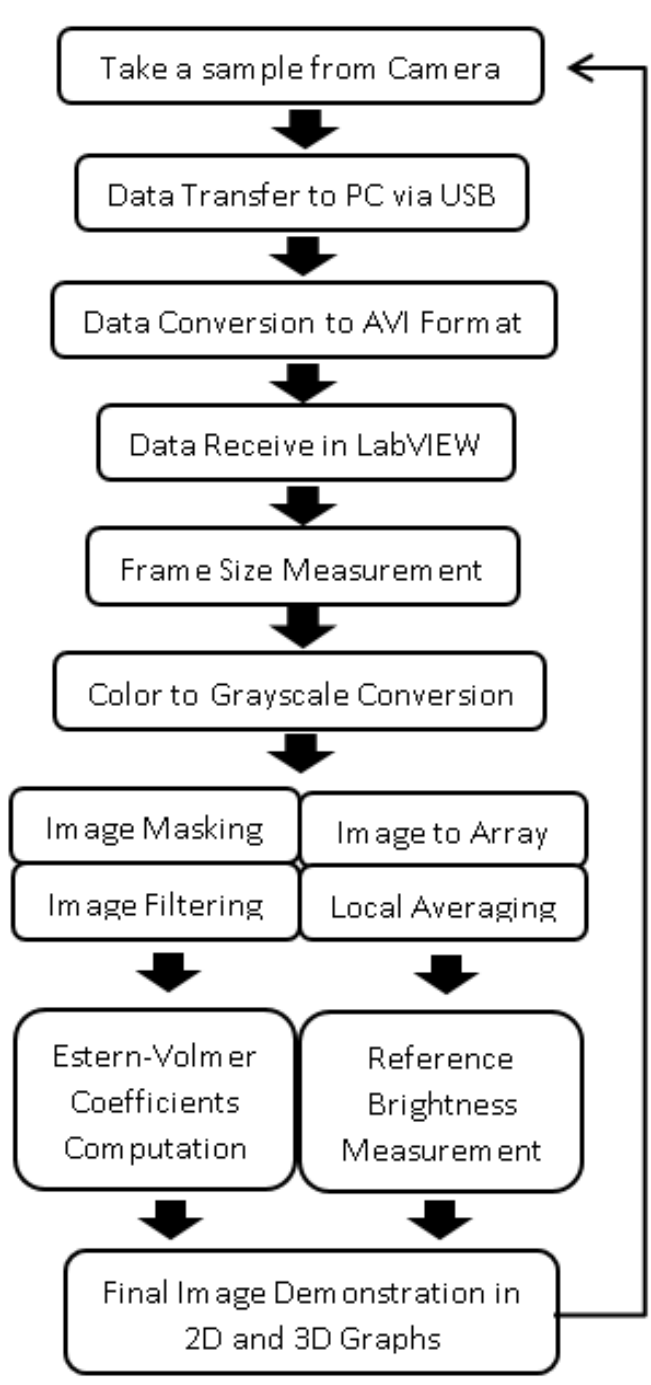

Fig. 4: Flowchart of image processing steps of the PSP surface pressure measurement system.
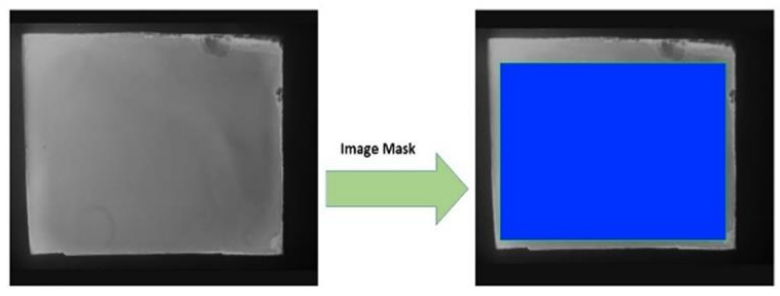

Fig. 5: Non-blue area on the right-hand side of the area removed from the image

One of the important parameters in color measurement of PSP pressure is the level of reference brightness. The reference brightness parameter is equal to the brightness of the sample surface at the initial test conditions, such as prior to changing the chamber or when the wind is not blowing in the wind 
tunnel "Wind off"[12]. The PSP test measures the brightness changes of the sample surface relative to the primary or reference illumination to minimize the effect of noise caused by non-uniform coatings and noise caused by the uniform illumination of the excitation lamp on the model. To calculate the surface brightness more accurately, it is better to measure the pixel surface brightness in seconds. This factor increases the accuracy of calibration as well as testing.

The range of changes per pixel for 8-bit cameras will be from 0 to 255. This is not enough to measure the amount of pressure changes. Suppose a test is to measure a range of pressure changes from 860 to 1100 mbar by pixel brightness changes, if the range of pixel brightness vary between 150 and 180 for this pressure range, then for a unit change in pixel value of 9 mbar will result in a change in the measured pressure that results in a low accuracy measurement. An example has been shown in Fig. 6.

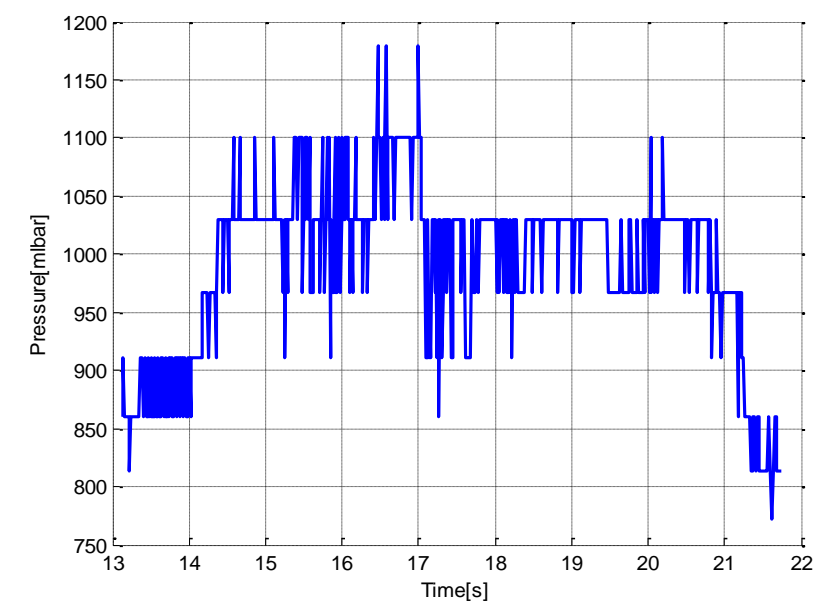

Fig. 6: Low resolution diagram of the pressure changes extracted from the 8-bit image.

\subsection{Local averaging}

In the process of calibration and development of the surface pressure measurement system, a method so called local averaging has been used to increase measurement accuracy. So, instead of measuring the whole number of pixels in the image, and considering every pixel as a sensor to measure the pressure changes, the averaged set of pixels has been taken as a pressure sensor cell. This means that the image is firstly transformed into an array of dimensions equal to the image resolution, and then the members of the array or pixels of the image, are divided into equal groups of array members. For instance, if there is an image of $640 \times 480$, it can be converted into a two dimensional array with the same dimensions, and divide the array members into $10 \times 10$ groups. Therefore, $64 \times 48$ groups of 100 members are obtained in the array. Next, the average value of every set of pixels or members is stored as a new member in another array, resulting in a new $64 \times 48$ image. In fact, instead of considering the individual pixels of the image as a sensor that represents the pressure value, the average set of 25 or 100 or higher values of 10000 can be considered as a sensor. This process reduces the noise significantly, and also increases the variation of the calculated pixels. In fact, the average value of the pixels calculated by this method is converted from the integer to the decimal number format, and this cause to reflects the variation accurately.

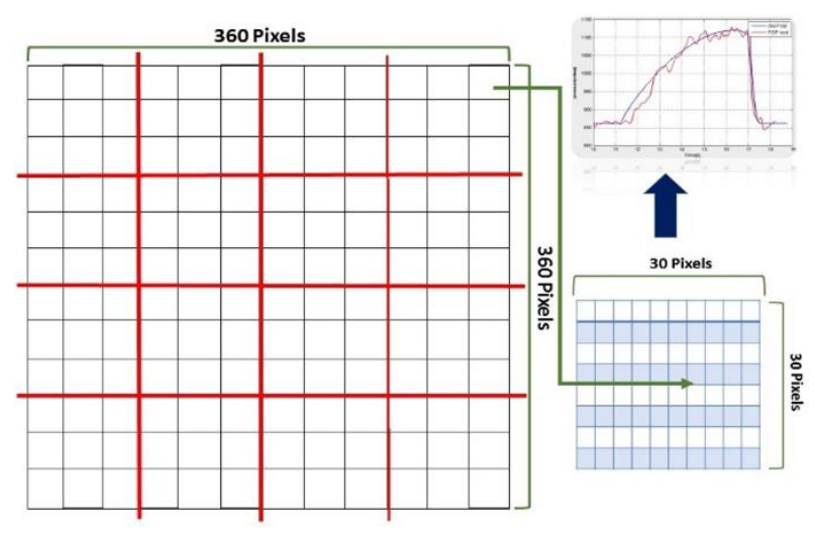

Fig. 7: shows an overview of how to implement local averaging on image pixels.

In fact, the averaging method can overcome the 8-bit resolution problem, and increase the range of variations due to increased number of averaged pixels. Fig.7 shows an overview of how pixels are grouped in the local averaging method.

\section{Calibration of surface pressure measurement system}

When performing the calibration procedure, two values must be measured simultaneously: 1) calibrated pressure sensor data and 2) Sample surface 
brightness variations computed by image processing. As the images are transmitted, the temperature and pressure data are transmitted to the computer by serial communication (COM), and then monitored by a $\mathrm{VI}^{1}$ in LabVIEW, and the pressure changes monitored online. Fig. 8 shows the laboratory set-up for calibration of the surface pressure measurement system.

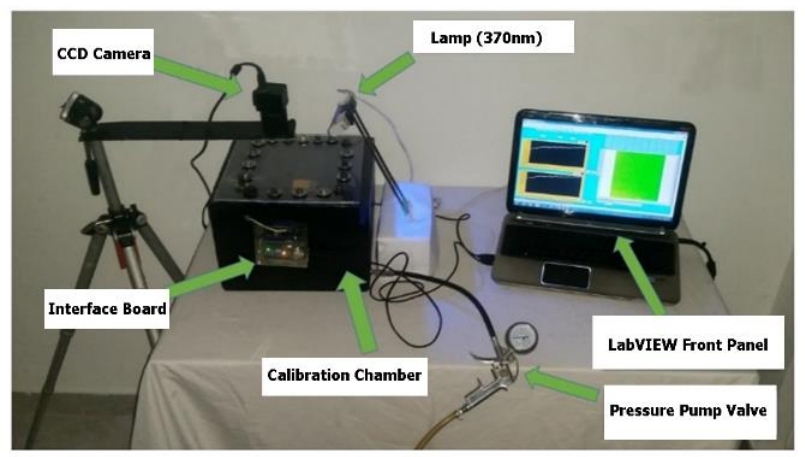

Fig. 8: Laboratory set-up for calibration of surface pressure measurement system

To start the test, connect the pump to the chamber, and start increasing pressure at a constant rate, and raise the pressure to 1100 mbar and stop. After completing this step, the user will be tested on two different output types: 1- Recorded image from sample surface 2- Diagram obtained from pressure variation measured by the benchmark sensor. The two types of the outputs are used to calculate the coefficients of the Estern-Volmer equation. These coefficients vary for different luminophore materials as well as different retaining layers. Though, by changing lighting conditions or other factors in the PSP test, these coefficients remain constant. In fact, by calculating the coefficients $\mathrm{A}$ and $\mathrm{B}$ during the calibration operation, the amount of pressure changes in the PSP test can be measured only by measuring the pressure changes on the surface without any other pressure sensors. The Estern-Volmer equation is expressed as follows. The coefficients $\mathrm{A}$ and $\mathrm{B}$ obtained for the pyrene material with the $\mathrm{TLC}^{2}$ retaining layer were calculated using the following equations. According to the basic test conditions:

\footnotetext{
${ }^{1}$ Virtual Instrument
}

$A+B=1$

Apply the end conditions according to the lighting diagram:

$$
1.0123 \times B+A=1.2746
$$

According to Equations (8) and (9), the coefficients $\mathrm{A}$ and $\mathrm{B}$ are calculated for the coating of the pyrene with the retaining layer.

$$
B=.0583 \quad A=.9417
$$

Now according to the obtained coefficients, it is possible to draw the pressure diagram measured by PSP method.

\section{Experimental Results}

To better compare and verify the accuracy of the coefficients obtained in the calibration, the diagram obtained from the BMP180 pressure sensor is plotted along with the mean value of the PSP method as shown in Fig. 9. In this test, the pressure has been decreased from $1100 \mathrm{mbar}$ to $870 \mathrm{mbar}$ (the air pressure at the test lab).

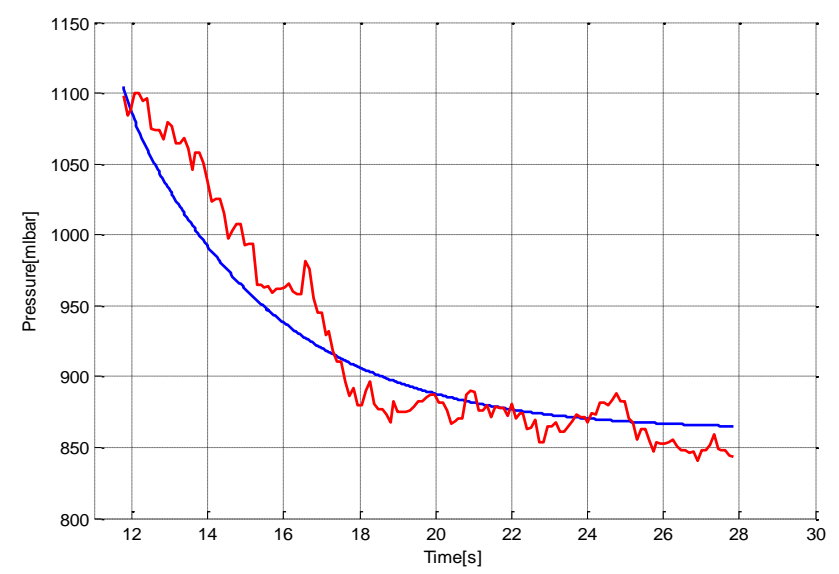

Fig. 9: Comparison of two graphs obtained from BMP180 pressure sensor (blue) and PSP test (red)

Fig. 10 shows the average 25-pixels variation profile of a 3-D image frame for the pyrene material. Surface

${ }^{2}$ Thin-layer chromatography 
pressure profiles over time are thus used in this analysis.

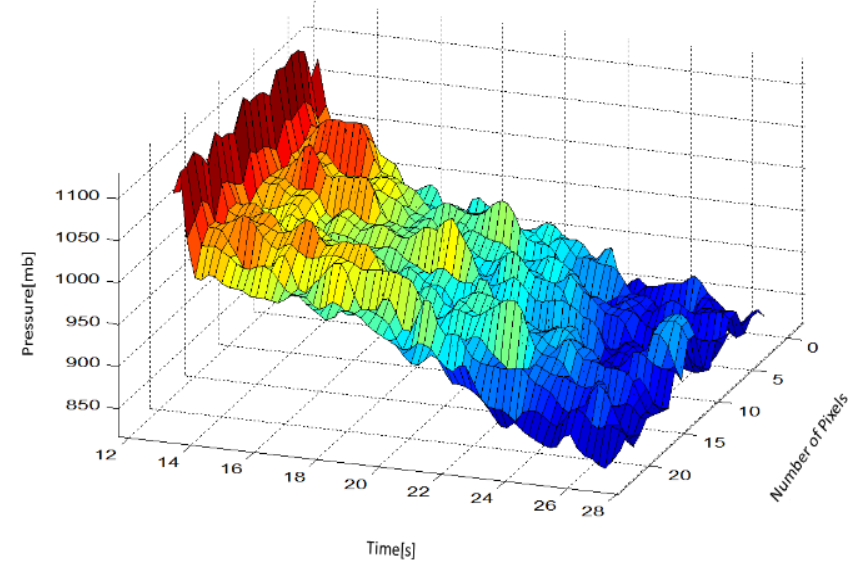

Fig. 10: Profiles of the average 25-pixel variations of a

3D image frame

Fig. 11 has been plotted after applying the frequency filter to the pressure measured by the PSP method. As can be seen, high frequency noise has been eliminated by applying low pass filter.

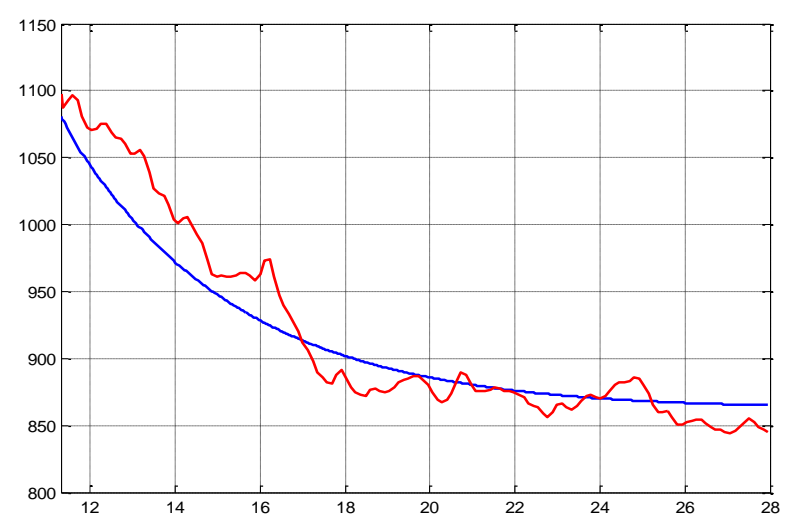

Fig. 11: Comparison of two graphs obtained from the BMP180 pressure sensor and PSP test averaged

In another experiment to investigate the temporal response of the PSP surface pressure measurement method, a pressure step was introduced into the chamber with a sudden increase in pressure to determine the extent of reaction of the coating to the changes. It should be noted that the high-pressure air from another high-pressure chamber has been entered to the test chamber via a pipe not in a really quick way. The test chamber pressure is discharged through the pipe and valve. it will take a short time for the air chamber pressure to equalize with the outside. Fig. 12 shows these changes. To better compare with the actual change diagram occurring in the system, both plots of calibrated pressure sensor (BMP180) and PSP test have been drawn simultaneously.

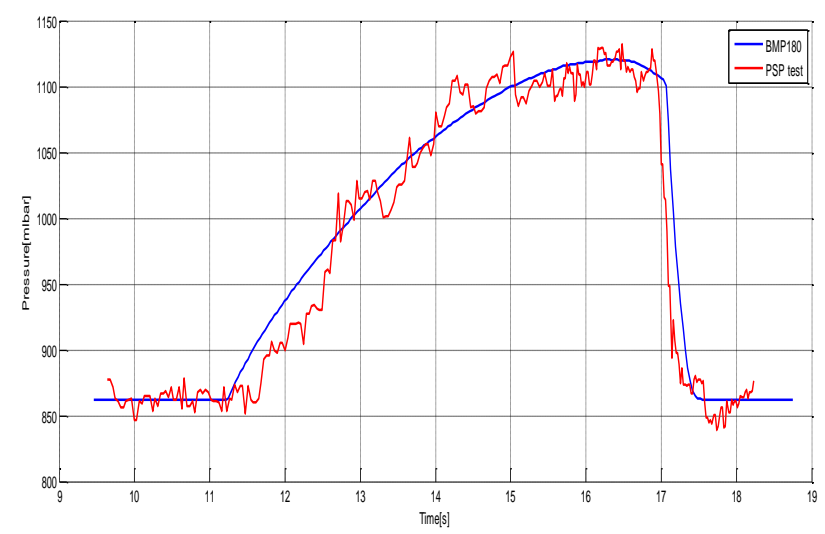

Fig. 12: Step Response of PSP Test Pressure (Blue), and Pressure Measured by BMP180 Sensor (Red)

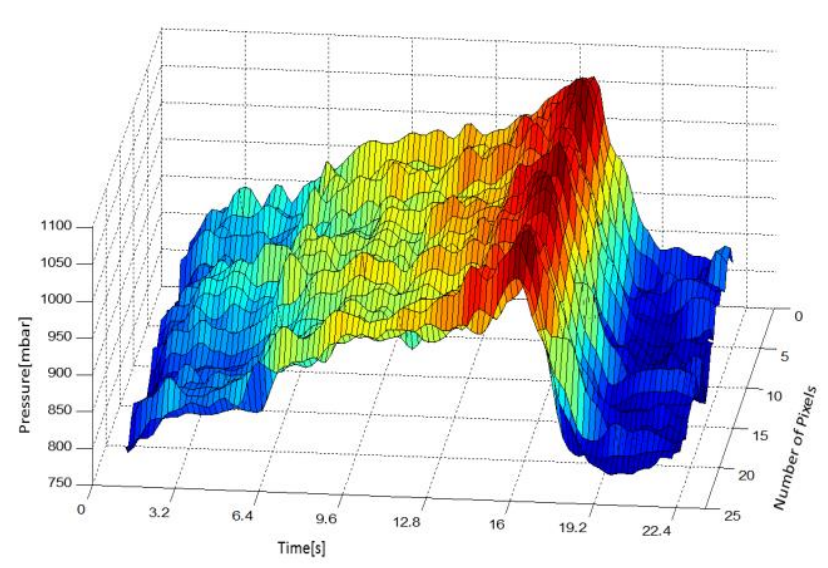

Fig. 13: 3D profile of how the sample responds to pressure changes for 25-pixels from the sample image

To better examine the variations of the pixels in the step test, a three-dimensional diagram containing a set of pixels with local averaging is plotted in Fig. 13. In this plot, the sample size is $360 \times 360$ pixels. In this graph, the sample size is $360 \times 360$ pixels, using a $72 \times 72$ pixels local averaging method as a sensor or pixel for measuring pressure. So, by applying this method, the number of pixels of the shape is changed to 25 pixels with much higher resolution that the 


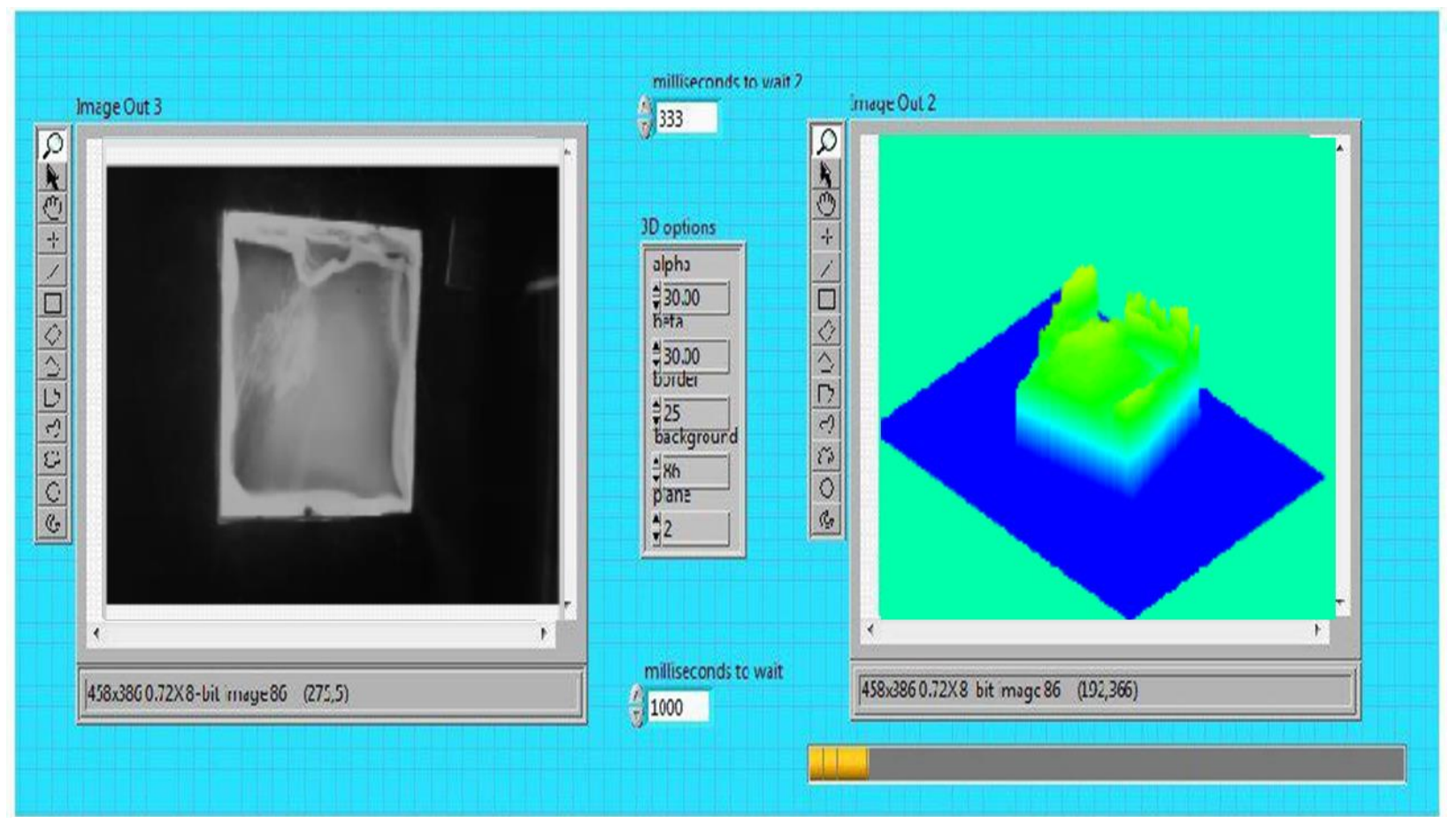

Fig. 14: Real-time image processing program control panel for displaying a surface pressure profile at $30 \mathrm{~S} / \mathrm{s}$

changes of every 25 pixels are plotted by applying step pressure to the chamber.

\subsection{Real-time processing of PSP pressure measurement images}

LabVIEW's PSP pressure gauge app is capable of measuring the level of pressure both online and offline based on the flowchart shown in Fig. 4. Realtime rules are possible for both application modes. To convert the image processing program to the realtime program initially, each program task must be separated by creating a Sub VI. The priority value of each Task should be determined by its degree of importance in the program. For example, Image Conversion Tasks and Calibration should have a higher priority than the Task Counting Camera Frame. For the real-time implementation of the algorithm a real-time hardware target that can run real-time LabVIEW Vis has been required. The realtime hardware are usually programmed and controlled by another computer called host computer. In this experiment, a MyRIO module has been used as the RT Target (target hardware for real-time application execution). Real-time rules has been fully utilized in image processing algorithm. The local averaging task was considered as the highest priority because of its higher sensitivity and its important role in the measurement. The difference in the results obtained from the real-time mode compared to the non-real-time mode was evident in the project. So that, the rate of pressure variations in response to the pressure measurement system was slower when no real-time laws were implemented, reaching a maximum of 20 samples per second $(20 \mathrm{~S} / \mathrm{s})$. After implementing the application in real-time and using the MyRIO module as the target hardware, these changes were made to 30 samples per second (30 $\mathrm{S} / \mathrm{s})$.

Fig. 14 shows the real-time image processing program panel under LabVIEW. In addition to adjusting different parameters, the program features the ability to change the viewing angle relative to the output chart $[14,15,16]$.

\subsection{Accuracy and precision of PSP pressure measurement system}

the accuracy is the most important feature of an instrument. The accuracy is calculated based on the maximum error of the device when displaying the results. Equation 10 shows how the error is calculated 
in percentage. $\mathrm{A}$ is the measured value and $\mathrm{B}$ is the true value.

$$
\% \mathrm{E}=\frac{\mathrm{A}-\mathrm{B}}{\mathrm{B}} \times 100
$$

Using the equation 10 and the three-dimensional graph (Fig. 13) and the two-dimensional graph (Fig. 12) obtained from the calibration, the maximum error is calculated as follows.

$$
\begin{gathered}
\mathrm{A}_{\mathrm{PSP}}=1050 . \mathrm{B}_{\mathrm{BMP} 180}=1000 \\
\mathrm{E}=\frac{50}{340} \times 100=14 \%
\end{gathered}
$$

Accuracy of measurement indicates the accuracy of the measured value with repeated measurement, which is closely related to the accuracy of the measurement. The accuracy of the measurement is obtained by examining the three-dimensional graph, and considering each pixel as a duplicate of the measurement. It can be concluded that the rate of error variations is between -40 and 50 . So, the Equation 13 shows:

$$
\operatorname{Precision}(\%)=\frac{90}{1010} \times 100 \cong 9 \%
$$

The accuracy of the results of the PSP surface pressure measurement method at $30 \mathrm{~S} / \mathrm{s}$ is about $9 \%$.

\section{Conclusion}

Development and implementation of a color pressure measurement system (PSP), provides a useful and powerful tool for designers to study complex airflow phenomena. This paper investigates the methods for real-time calibration of a PSP surface pressure measurement system using pyrene luminophore material. The results are used to calibrate the measurement system to obtain the $\mathrm{A}$ and $\mathrm{B}$ coefficients of the Estern-Volmer equation using a calibrated pressure sensor. The results obtained from different experiments show that the pressure measurement at any point of the surfaces performed by PSP method and appropriate luminophore material has acceptable accuracy. Typical image sampling and processing speed with a desktop computer is about $20 \mathrm{~S} / \mathrm{s}$, but when a real-time hardware is used for image processing and the real- time image processing algorithm has been employed, the sample rate increased to $30 \mathrm{~S} / \mathrm{s}$. The hardware part of the calibration system includes the CCD camera, calibration chamber with controlled valves, a lamp with 370nm wavelength, a calibrated pressure sensor and a MyRIO module for real-time image processing. The software part has been implemented in the LabVIEW graphical environment in real-time. A control panel is designed to work with software that provides designers with various information on how the pressure has been distributed in 3-D and 2-D color charts.

\section{References}

[1] M. D. Rockwell, Pressure and Temperature Sensitive Paints. 2005.

[2] R. Blades et al., "Development of a Pressure Sensitive Paint System for Measuring Global Surface Pressures on," 2007.

[3] L. Yang, E. Erdem, H. Zare-Behtash, K. Kontis, and S. Saravanan, "Pressure-sensitive paint on a truncated cone in hypersonic flow at incidences," Int. J. Heat Fluid Flow, vol. 37, pp. 9-21, 2012.

[4] D. Zur and E. Des, "Preparation and Calibration of Pressure- Sensitive and Temperature Sensitive Paints for Fluorescence Lifetime Imaging Applications," 2009.

[5] H. Sakaue and K. Ishii, "Optimization of Anodized-Aluminum Pressure-Sensitive Paint by Controlling Luminophore Concentration," no. Fig. 1, pp. 6836-6847, 2010.

[6] Y. Egami, Y. Matsuda, H. Yamaguchi, and T. Niimi, "Sensors and Actuators B: Chemical Property changes of temperature-sensitive paint immobilized in acrylic polymer matrices," Sensors Actuators B. Chem., vol. 195, pp. 677681, 2014.

[7] M. For and G. Vision, "Basler pilot," vol. 0, no. March, 2013.

[8] B. Sensortec, "BMP180 Datasheet." Oct, 2014.

[9] N. I. L. U. Manual, National Instruments (2003). 2015.

[10] A. C. Bovik, The essential guide to image processing. Academic Press, 2009.

[11] R. Posada-Gómez, O. O. SandovalGonzález, A. M. Sibaja, O. Portillo-Rodríguez, and G. Alor-Hernández, "Digital Image Processing Using LabView," in Practical Applications and Solutions Using LabVIEW ${ }^{\mathrm{TM}}$ Software, InTech, 2011.

[12] K. Kontis and U. K. M, "Experimental Studies on the Effects of Thermal Bumps in the 
Flow-Field around a Flat Plate using a Hypersonic Wind Tunnel University of Manchester Report Date: APO AE 09421," no. January, 2012.

[13] Y. Angal and A. Gade, "LabVIEW controlled robot for object handling using NI myRIO," 2016 IEEE Int. Conf. Adv. Electron. Commun. Comput. Technol. ICAECCT 2016, pp. 167-171, 2017.

[14] T. H. Drayer, W. E. King, J. G. Tront, R. W. Conners, and P. A. Araman, "Using multiple FPGA architectures for real-time processing of low-level machine vision functions," in Industrial Electronics, Control, and Instrumentation, 1995., Proceedings of the 1995 IEEE IECON 21st International Conference on, 1995, vol. 2, pp. 1284-1289.

[15] A. C. Bovik, The essential guide to image processing. Academic Press, 2009.

[16] R. Posada-gómez, O. O. Sandoval-González, A. Martínez Sibaja, O. Portillo-Rodríguez, and G. Alor-Hernández, "Digital Image Processing Using LabView," Intechopen, 2001.

\section{Authors}

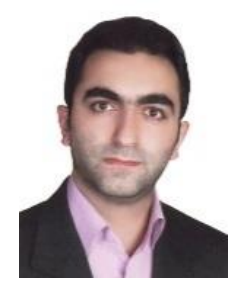

Mohsen Davoudi received his Ph.D. in Electrical Engineering from Polytechnic University of Milan (Politecnico di Milano), Milan, Italy, in 2011. Currently he has assistant professor position at Imam Khomeini Qazvin, Iran. International University (IKIU),

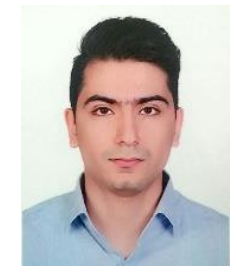

Afshin Heidarian received his BSc degree in Electrical Engineering from Shiraz University in 2015. He is presently pursuing Masters in Electrical Engineering at Imam Khomeini International University, Qazvin, Iran. His research interests are Image processing and enhancement, Hardware implementation of controllers (fuzzy, adaptive, PID...) and Instrumentation. 